\title{
Pacific
}

Journal of

Mathematics

\section{MINIMAL SURFACES WITH CATENOID ENDS}

JORGEN BERGLUND AND WAYNE ROSSMAN 


\title{
MINIMAL SURFACES WITH CATENOID ENDS
}

\author{
JoRgen Berglund AND Wayne Rossman
}

\begin{abstract}
A substantive part of the recent activity in the field of minimal surface theory has been the construction of new complete minimal surfaces immersed in $\mathbb{R}^{3}$. One approach in constructing new examples is to increase the genus of known minimal surfaces. In this paper, we do precisely this for certain minimal surfaces of finite total curvature whose ends are asymptotic to catenoids. We prove existence of surfaces of positive genus based on those in genus zero, with the feature that these higher genus examples maintain all the symmetry of their genus-zero counterparts. In these proofs we use the conjugate minimal surface construction and the maximum principle for minimal surfaces.
\end{abstract}

\section{Introduction}

In the last century, O. Bonnet, and later H. A. Schwarz, were the first to study the associate family of a minimal surface ([Ni2], [Scz $])$. More recently, A. Schoen, H. Karcher, and others have used properties of the associate family to develop a method for constructing periodic minimal surfaces ([Ka1], [Ka2], [Ka3], [Ka4], [Kr]). This method uses the particular member of the associate family known as the conjugate surface, and is referred to, by Karcher, as the Conjugate Plateau Construction. W. H. Meeks III suggested using this construction to study non-periodic examples ([Me]).

This construction is used to prove the results here. The technique begins by considering the boundary contour of the conjugate of a fundamental piece of the surface. The contour can be described as partially unbounded boundary data over an unbounded convex domain. We extend results of J. C. C. Nitsche [Ni1] and Jenkins and Serrin [JeSe] to this setting and obtain the existence of a unique minimal surface with this given boundary. The existence of the original surface can then be argued.

Our main results concern the existence of immersed finite-total-curvature minimal surfaces with embedded catenoid ends and genus greater than zero:

1) For each $n \geq 3$, there exists an $n$-oid of genus 1 that maintains all the symmetry of the genus-0 $n$-oid (see Figures 1.1 and 1.2). 

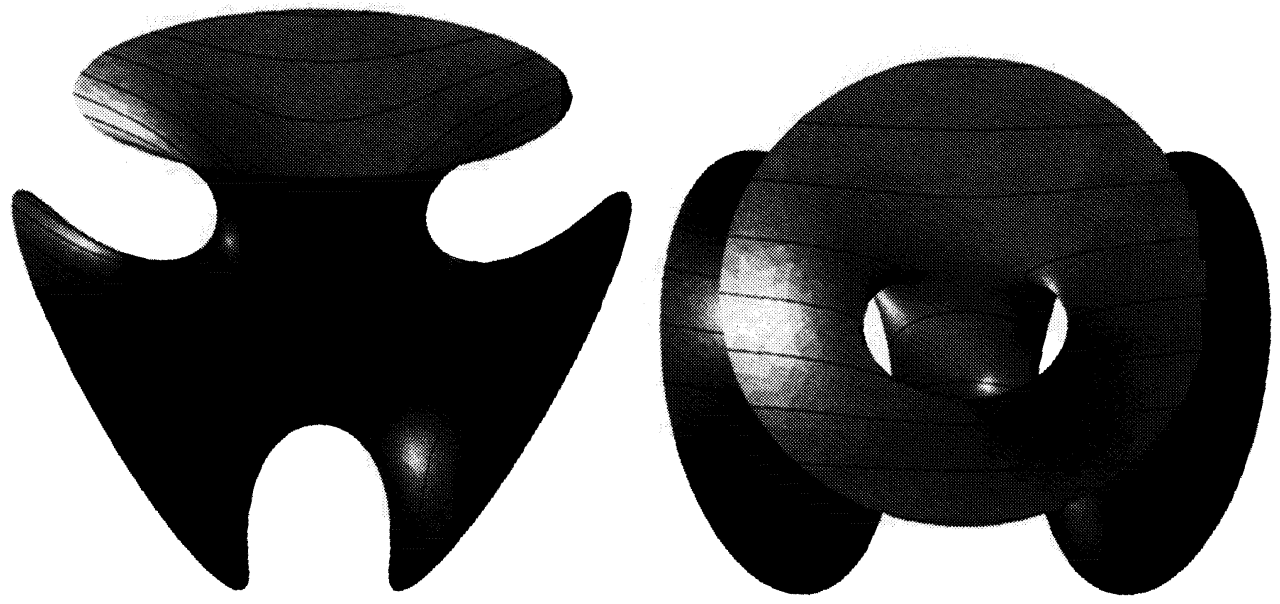

Figure 1.1: The trinoid

2) There exist minimal surfaces based on each of the Platonic solids. These surfaces are of genus $f-1$ and have $v$ catenoid ends, where $f$ and $v$ are the number of faces and vertices of the corresponding Platonic solid (see Figure 4.13).

We also prove the nonexistence of a certain symmetric $n$-oid of genus $n$, while indicating why another type might exist (see Figures 3.10 and 3.11). Throughout the paper, we use Weierstrass data to draw the surfaces with computer graphics [MESH], and we conclude this paper by deriving Weierstrass data for the trinoid of genus 1 . These data yield numerical evidence for the existence of less symmetric examples (see Figure 4.17).

The authors wish to thank Rob Kusner for many helpful suggestions and critical readings of preliminary drafts. We would also like to thank: Fusheng Wei for assistance in deriving Weierstrass data; Martin Traizet for creating Figures 3.11 and 4.13; and David Hoffman, Ed Thayer, and others at G.A.N.G. for helpful conversations and assistance with computer graphics.

\section{Tools}

The following tools will be used extensively in the subsequent proofs.

2.1. Conjugate Surface Construction for Minimal Surfaces. For an immersed minimal surface $M$ in $\mathbb{R}^{3}$ with finite total curvature, there exists a meromorphic function $g$ and a holomorphic 1-form $\eta$ defined on a punctured compact Riemann surface $\bar{M} \backslash\left\{p_{1}, p_{2}, \ldots, p_{\ell}\right\}$ such that $M$ has the 

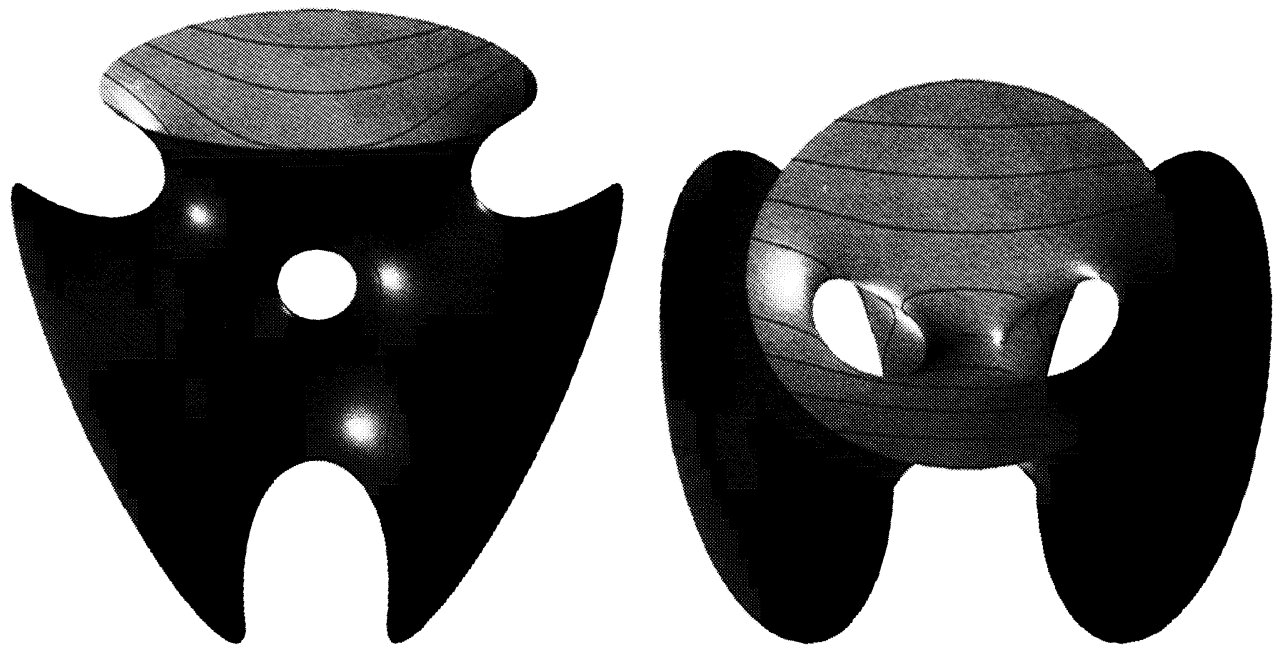

Figure 1.2: The symmetric trinoid of genus 1

parametrization

$$
\Phi(p)=\operatorname{Re} \int_{p_{0}}^{p}\left(\begin{array}{c}
\left(1-g^{2}\right) \eta \\
i\left(1+g^{2}\right) \eta \\
2 g \eta
\end{array}\right), \quad p \in \bar{M} \backslash\left\{p_{1}, p_{2}, \ldots, p_{\ell}\right\}
$$

We refer to $\{g, \eta\}$ as the Weierstrass data for $M$, and to $\Phi$ as the Weierstrass representation of $M$. The conjugate surface $M_{\text {conj }}$ of $M$ is the minimal surface with the same underlying Riemann surface $\bar{M} \backslash\left\{p_{1}, p_{2}, \ldots, p_{\ell}\right\}$, but with Weierstrass data $\{g, i \eta\}$. Strictly speaking, the parametrization $\Phi_{\text {conj }}(p)$ may only be well-defined on a covering of $\bar{M} \backslash\left\{p_{1}, p_{2}, \ldots, p_{\ell}\right\}$.

Thus we have the maps $z \rightarrow \Phi(z)$ and $z \rightarrow \Phi_{\text {conj }}(z)$ from simply connected domains of $\bar{M} \backslash\left\{p_{1}, p_{2}, \ldots, p_{\ell}\right\}$ to $M$ and $M_{\text {conj }}$, respectively. This induces a covering map $\phi$, the conjugate map, from $M_{\text {conj }}$ to $M$. The conjugate map $\phi$ has the following properties:

1) $\phi$ is an isometry;

2) $\phi$ preserves the Gauss map;

3) $\phi$ maps planar principal curves in $M_{\text {conj }}$ to planar asymptotic curves in $M$, and maps planar asymptotic curves in $M_{\text {conj }}$ to planar principal curves in $M$; that is to say, $\phi$ maps non-straight planar geodesics to straight lines, and vice versa.

It follows from the second and third properties of $\phi$ that a planar geodesic is mapped by $\phi$ to a line that must be perpendicular to the plane containing the planar geodesic.

We note that the conjugate of the conjugate of $M$ is given by the Weierstrass data $\{g,-\eta\}$, locally giving us the original surface reflected through 


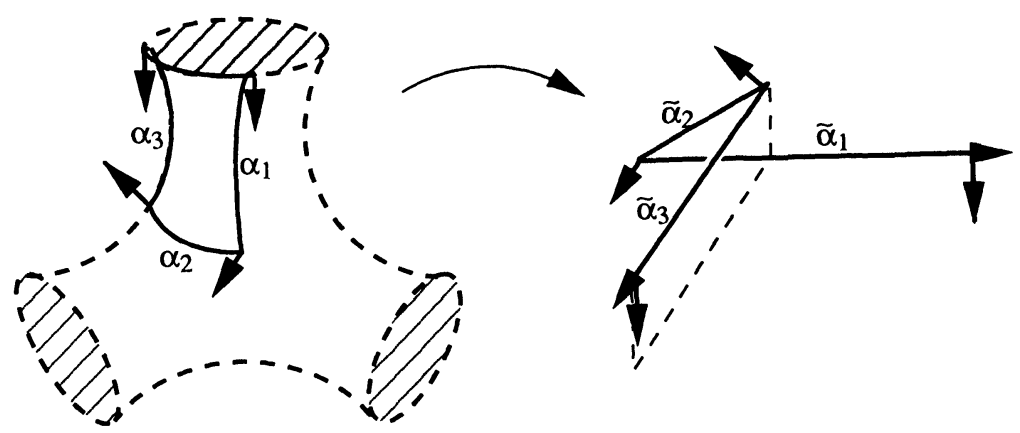

Figure 2.3: The fundamental piece of a trinoid, and its conjugate surface.

the origin.

Example. A fundamental piece of a minimal surface is a smallest portion of the surface that can generate the entire surface when acted upon by the surface's symmetries. Consider a fundamental piece of an $n$-oid. $4 n$ copies of this fundamental piece are needed to create the entire surface. Note that the boundary of this fundamental piece is composed entirely of planar geodesics. The conjugate surface of this fundamental piece is thus easily determined by considering the properties above. It is a graph over the interior of an unbounded convex region in some plane and thus is simply connected. This region is bounded by two parallel infinite rays and one line segment connecting the endpoint of each ray (see Figure 2.3).

We shall say that a minimal surface has a helicoid end if the corresponding end of the conjugate surface is a portion of a catenoid end. Thus, in the example above, the conjugate surface of a fundamental piece of the $n$-oid has a helicoid end.

2.2. The Maximum Principle for Minimal Surfaces The following well-known lemma is the maximum principle for minimal surfaces. It is a special case of a lemma by Schoen [Scn], and is proven there.

Lemma 2.1. 1) (Interior Maximum Principle). Let $M_{1}$ and $M_{2}$ be minimal surfaces in $\mathbb{R}^{3}$. Suppose $p$ is an interior point of both $M_{1}, M_{2}$, and suppose $T_{p}\left(M_{1}\right)=T_{p}\left(M_{2}\right)$. If $M_{1}$ lies on one side of $M_{2}$ near $p$, then $M_{1}=M_{2}$.

2) (Boundary Point Maximum Principle). Suppose $M_{1}, M_{2}$ have $: C^{2}$ boundaries $C_{1}, C_{2}$, respectively, and suppose $p$ is a point of both $C_{1}, C_{2}$. Furthermore, suppose the tangent planes of both $M_{1}, M_{2}$ and $C_{1}, C_{2}$ agree at $p$ : that is to say, suppose $T_{p}\left(M_{1}\right)=T_{p}\left(M_{2}\right), T_{p}\left(C_{1}\right)=T_{p}\left(C_{2}\right)$. If, near $p$, $M_{1}$ lies to one side of $M_{2}$, then $M_{1}=M_{2}$. 
2.3. Results on the Existence of Minimal Surfaces. The following are two existence theorems. The first theorem is due to Nitsche ([Ni1], [JeSe]), and the second is due to Jenkins and Serrin ([JeSe]).

Theorem 2.2. Let $D$ be a bounded convex domain in a plane. Let $\partial \tilde{D}=$ $\partial D \backslash\left\{p_{1}, \ldots, p_{r}\right\}$. Then there exists a solution of the minimal surface equation in $D$ taking on preassigned bounded continuous data on the arcs of $\partial \tilde{D}$. As a surface, this solution contains vertical line segments over the jump discontinuities of the boundary data.

Theorem 2.3. Monotone convergence theorem: Let $\left\{M_{n}\right\}_{n=1}^{\infty}$ be a monotone increasing sequence of solutions of the minimal surface equation in a domain $D$. If the sequence is bounded at a single point $p \in D$, then there exists a nonempty open set $U \subseteq D$ such that $\left\{M_{n}\right\}_{n=1}^{\infty}$ converges to a solution in $U$, and diverges to infinity on the complement of $U$. The convergence is uniform on compact subsets of $U$.

\section{Adding Handles to the $n$-oid}

The $n$-oids are well-known immersed genus- 0 minimal surfaces of finite total curvature in $\mathbb{R}^{3}$ (see Figure 1 and [JoMe]). Their defining feature is that they have $n$ catenoid ends, whose limiting normals span a plane $\mathcal{P}$, which is a plane of reflective symmetry of the surface. In addition, $n$-oids have a degree $n$ rotational symmetry about an axis perpendicular to $\mathcal{P}$ and a plane of reflectional symmetry also perpendicular to $\mathcal{P}$. Thus the symmetry group of an $n$-oid is $D_{n} \times \mathbb{Z}_{2}$, the natural $\mathbb{Z}_{2}$-extension of the dihedral group.

In this section, we consider the problem of adding $k$ handles to the $n$-oid, while preserving minimality. We refer to these as $n$-oids of genus $k$.

Theorem 3.1. For each $n \geq 3$, there exists an $n$-oid of genus 1 that maintains all the symmetries of the genus-0 n-oid.

Proof. We approach the proof in the following manner: If the genus 1 surface exists, then it has a simply connected fundamental piece and the conjugate of this fundamental piece must also exist. The boundary contour of this conjutgate piece is among a 1-parameter family $\hat{C}_{\lambda}$ of contours, each of which, we show, bounds an embedded simply connected minimal surface. The original fundamental piece is, up to congruence, the conjugate to one of these, for a particular choice of $\lambda$ which "kills the period". That is to say, $\lambda$ is chosen so that the original fundamental piece extends by reflection and rotation to the conjectured surface. We show that $n \geq 3$ is precisely the necessary and sufficient condition for solving this period problem. 


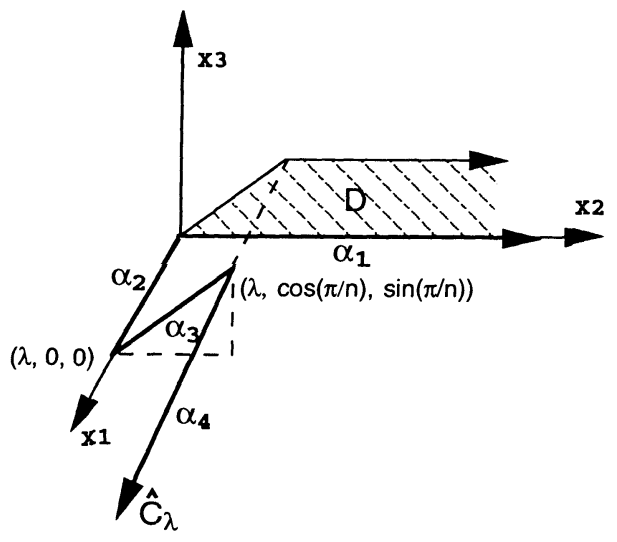

Figure 3.4: The contour $\hat{C}_{\lambda}$ and the convex planar domain $D$.

For any $\lambda \geq 0$ define a contour $\hat{C}_{\lambda}$ in $\mathbb{R}^{3}$ consisting of two straight rays and two line segments. Let $\alpha_{1}$ be the ray $\{(0, s, 0): s \geq 0\}$; let $\alpha_{2}$ be the line segment with endpoints at $(0,0,0)$ and $(\lambda, 0,0)$; let $\alpha_{3}$ be the line segment with endpoints at $(\lambda, 0,0)$ and $\left(\lambda, \cos \left(\frac{\pi}{n}\right), \sin \left(\frac{\pi}{n}\right)\right)$; and let $\alpha_{4}$ be the ray $\left\{\left(\lambda+s, \cos \left(\frac{\pi}{n}\right), \sin \left(\frac{\pi}{n}\right)\right): s \geq 0\right\}$; then $\hat{C}_{\lambda}$ is $\alpha_{1} \cup \alpha_{2} \cup \alpha_{3} \cup \alpha_{4}$. We note that the projection of $\hat{C}_{\lambda}$ to the plane $\left\{x_{1}=0\right\}$ lies in the boundary of an unbounded convex domain, which we will call $D$ (see Figure 3.4).

The contour $\hat{C}_{0}$ is the boundary of the conjugate surface of the fundamental piece of the $n$-oid. Translating $\hat{C}_{0}$ in the $x_{1}$-direction by $(\lambda, 0,0)$ we have a contour that we shall call $\hat{C}_{\text {trans }, \lambda}$. (Note that $\hat{C}_{0}=\hat{C}_{\text {trans }, 0}$.) It follows from the known existence of the $n$-oid of genus 0 that, for all $\lambda$, the contour $\hat{C}_{\text {trans }, \lambda}$ bounds a minimal surface $\hat{M}_{\text {trans }, \lambda}$. The interior of $\hat{M}_{\text {trans }, \lambda}$ is a graph over the interior of the domain $D$.

We shall use the surface $\hat{M}_{0}=\hat{M}_{\text {trans }, 0}$ to construct compact contours which converge to $\hat{C}_{\lambda}$. Note that $\hat{C}_{0}$ coincides with $\hat{C}_{\lambda}$ along $\alpha_{1}$ and $\alpha_{4}$. Choose a strictly increasing sequence $\lambda_{\imath} \in \mathbb{R}$ such that $\lambda_{0}>\lambda$ and $\lim _{i \rightarrow \infty} \lambda_{i}=$ $\infty$. For each $\lambda_{i}$, choose a curve $\gamma_{i}$ lying in $\hat{M}_{0}$ with the following properties:

1) $\gamma_{\imath}$ has endpoints $\left(\lambda_{2}, \cos \left(\frac{\pi}{n}\right), \sin \left(\frac{\pi}{n}\right)\right)$ and $\left(0, \lambda_{\imath}, 0\right)$.

2) $\gamma_{i}$ projects onto a curve $\operatorname{proj}\left(\gamma_{i}\right)$ in $D$ which is convex with respect to the bounded component of $D \backslash \operatorname{proj}\left(\gamma_{i}\right)$.

3) $\gamma_{\imath} \cap \gamma_{j}=\phi$ for all $i \neq j$.

Let $C_{\lambda_{2}}$ be the compact contour constructed by truncating the two rays $\alpha_{1}$, $\alpha_{4}$ of $\hat{C}_{\lambda}$ at $\left(\lambda_{i}, \cos \left(\frac{\pi}{n}\right), \sin \left(\frac{\pi}{n}\right)\right)$ and $\left(0, \lambda_{i}, 0\right)$, and then joining these endpoints by the curve $\gamma_{i}$. The contour $C_{\lambda_{2}}$ projects to a convex plane curve. Let $D_{i}$ be the bounded convex region with boundary consisting of this projection. $D_{i} \subseteq D$, and $\lim _{i \rightarrow \infty} D_{i}=D$ (see Figure 3.5).

Essentially $\left\{\gamma_{i}\right\}_{i=0}^{\infty}$ is a sequence of curves in $\hat{M}_{0}$ so that, for $j>i, \gamma_{j}$ lies "farther out" on the end of $\hat{M}_{0}$ than $\gamma_{2}$. Since $\hat{M}_{0}$ is a graph over $D$, we 


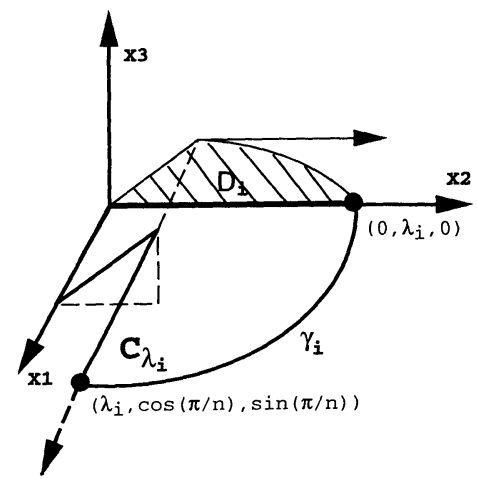

Figure 3.5: The curve $\gamma_{i}$, the contour $C_{\lambda_{i}}$, and the bounded planar region $D_{i}$.

conclude that $D_{i} \subseteq D_{j}$ for $j>i$. Furthermore, since $\lambda_{i} \rightarrow \infty$ and $\gamma_{i}$ projects to a convex curve in $D$, we may conclude that $\gamma_{i}$ lies outside any given compact region in $\mathbb{R}^{3}$, for $i$ chosen large enough. Thus, $\lim _{i \rightarrow \infty} C_{\lambda_{i}}=\hat{C}_{\lambda}$.

Since $C_{\lambda_{i}}$ can be viewed as piece-wise continuous boundary data on the domain $D_{i}$, discontinuous at only two points, we can apply Theorem 2.1. Therefore, for each $i$, there exists a minimal surface bounded by $C_{\lambda_{i}}$. Applying the maximum principle, we see that these solutions are unique, and we call these unique solutions $M_{\lambda_{i}}$. For any fixed $i$, the surface $M_{\lambda_{j}}$, for $j \geq i$, restricts to a solution of the minimal surface equation over $D_{i}$. Note that the restriction of $M_{\lambda}$, to $D_{i}$ may have different boundary data than $M_{\lambda_{i}}$ over $D_{i}$.

Claim 3.2. Fixing a positive integer $i$, the restrictions of $M_{\lambda}$, to $D_{i}$, for $j \geq i$, form a monotonically increasing sequence of solutions of the minimal surface equation over $D_{i}$.

Proof. Let $\epsilon$ be a fixed positive number. By "sliding $M_{\lambda_{k}}$ underneath $M_{\lambda_{j}}$ ", for $i \leq k<j$, we mean this: We start with copies of $M_{\lambda_{k}}$ translated by the vectors $s \cdot(-1,0,+\epsilon)$ for $s \geq 0$, and we call these copies $M_{\lambda_{k}, s}$. Since $M_{\lambda_{k}}$ and $M_{\lambda_{j}}$ are both graphs over $D$, it is clear that for $s>\frac{1}{\epsilon} \sin \left(\frac{\pi}{n}\right), M_{\lambda_{k}, s}$ and $M_{\lambda_{j}}$ are disjoint. Choose $s>\frac{1}{\epsilon} \sin \left(\frac{\pi}{n}\right)$. We then lower the value of $s$ until we reach the first value of $s$ so that $M_{\lambda_{k}, s} \cap M_{\lambda_{1}} \neq \phi$ (see Figure 3.6). Let $s_{0}=\sup \left\{s \geq 0 \mid M_{\lambda_{k}, s} \cap M_{\lambda_{3}} \neq \phi\right\}$. Proving the claim is equivalent to showing that $s_{0}=0$. Thus, we are sliding one surface underneath the other with respect to the positive $x_{1}$ direction.

In all subsequent references to height, we mean height with respect to the positive $x_{1}$ direction. (In Figure 3.6, we see $M_{\lambda_{j}}$ and a translated copy of $M_{\lambda_{2}}$. From the point of view of the positive $x_{3}$-axis, the copy of $M_{\lambda_{i}}$ lies above $M_{\lambda_{j}}$. But with respect to the positive $x_{1}$-axis, the copy of $M_{\lambda_{i}}$ 


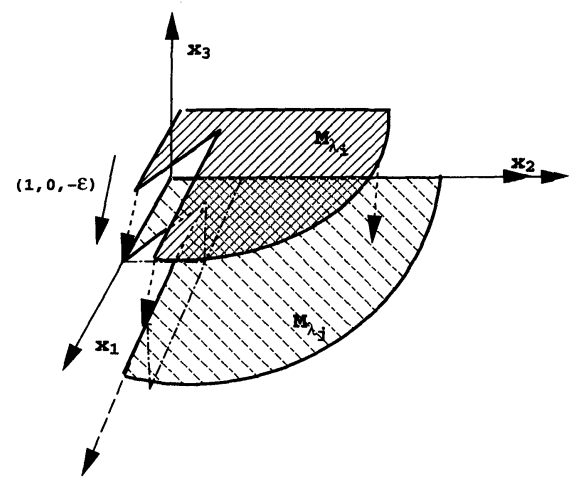

Figure 3.6: The surface $M_{\lambda_{k}}$ sliding "underneath" $M_{\lambda_{j}}$.

actually lies below $M_{\lambda_{j}}$. We take the latter perspective here.)

By the interior maximum principle, this first contact between $M_{\lambda_{k}, s}$ and $M_{\lambda_{j}}$ occurs along the boundary of $M_{\lambda_{k}, s}$. The first point of contact cannot occur at a point of the translated copy of $\gamma_{k}$. We can see this by showing that the original copy of $\gamma_{k}$ must lie strictly to one side of $M_{\lambda_{j}}$, except at its endpoints. The curve $\gamma_{k}$ is, by construction, a curve on $\hat{M}_{0}$. Again using the interior maximum principle, the surface $\hat{M}_{0}$ can be "slid underneath" $M_{\lambda_{j}}$ with no first point of contact in the interior. In particular, there is no first point of contact on the interior of $\gamma_{k}$, and we conclude $\gamma_{k}$ lies underneath $M_{\lambda_{j}}$. Thus the contact between $M_{\lambda_{k}, s_{0}}$ and $M_{\lambda_{j}}$ occurs in $\partial M_{\lambda_{k}, s_{0}}$, but cannot occur within the interior of the translated copy of $\gamma_{k}$ in $\partial M_{\lambda_{k}, s_{0}}$. Hence, the contact between $M_{\lambda_{j}}$ and $M_{\lambda_{k}, s_{0}}$ must occur along the common straight line boundaries of the two surfaces, and so $s_{0}$ must be zero. Therefore $M_{\lambda_{k}}$ lies entirely underneath $M_{\lambda_{j}}$. This proves the claim.

In the proof of the claim above, if we replace " $M_{\lambda_{k}}$ " by " $M_{\lambda_{j}}$ ", and we replace " $M_{\lambda_{j}}$ " by " $\hat{M}_{\text {trans, },}$ ", we have a proof that $M_{\lambda_{j}}$, for any $j$, lies underneath $\hat{M}_{\text {trans, } \lambda}$. Thus, over any point $p \in D_{i}$, the height of the solutions $M_{\lambda_{j}}$ are uniformly bounded above by the height of $\hat{M}_{\lambda}$ over $p$. This allows us to apply the monotone convergence theorem.

By Theorem 2.2, since the solutions $M_{\lambda_{j}}, j \geq i$, are monotonically increasing and are uniformly bounded-above over each $p \in D_{i}$, we conclude that the sequence of solutions $\left\{M_{\lambda_{j}}\right\}_{j=i}^{\infty}$, restricted to the domain $D_{i}$, converges uniformly to some solution over $D_{i}$. Since the choice of $i$ is arbitrary and $\lim _{i \rightarrow \infty} D_{\lambda_{i}}=D$, we see that the sequence of solutions $\left\{M_{\lambda_{j}}\right\}_{j=1}^{\infty}$ converges to a solution over $D$, and we call this solution $\hat{M}_{\lambda}$. Considered as a surface, $\hat{M}_{\lambda}$ has boundary $\hat{C}_{\lambda}$, and is a graph over $D$.

Let $\tilde{M}_{\lambda}$ be the conjugate surface of $\hat{M}_{\lambda}$. Denote the boundary of $\tilde{M}_{\lambda}$ by $\tilde{C}_{\lambda}$, and denote each planar geodesic in $\tilde{C}_{\lambda}$ by $\tilde{\alpha}_{i}$, in correspondence with its preimage line segment or ray $\alpha_{i} \subseteq \hat{C}_{\lambda}$ (see Figure 3.7). 
Note that the surfaces $\hat{M}_{\text {trans, } \lambda}$ and $\hat{M}_{0}$ both have helicoid ends, which are asymptotic to each other. Since $\hat{M}_{\lambda}$ lies between these two surfaces, the asymptotic behavior of the end of $\hat{M}_{\lambda}$ is determined. We now argue that $\hat{M}_{\lambda}$ has a helicoid end. We do this by showing that $\hat{M}_{\lambda}$ has finite total curvature (thus $\tilde{M}_{\lambda}$ has finite total curvature), and then applying Schoen's result on complete finite-total-curvature ends [Scn].

Choose an orientation on $M_{\lambda_{2}}$, and consider the Gauss map $G: M_{\lambda_{i}} \rightarrow S^{2}$. Since $M_{\lambda_{i}}$ is a graph, the image $\operatorname{Im}\left(M_{\lambda_{2}}\right) \subseteq S^{2}$ of $M_{\lambda_{2}}$ under $G$ must be contained in a hemisphere. The image $\operatorname{Im}\left(C_{\lambda_{i}}\right)$ of $C_{\lambda_{2}}=\partial M_{\lambda_{2}}$ under $G$ is a set of curves in $S^{2} . M_{\lambda_{2}}$ is a compact surface, hence has finite total curvature. Therefore $G$ is a branched covering map from $M_{\lambda_{2}}$ to $\operatorname{Im}\left(M_{\lambda_{2}}\right)$ with finite degree.

Let $P$ be the plane containing the points $(0,0,0),(\lambda, 0,0)$, and $\left(\lambda, \cos \left(\frac{\pi}{n}\right)\right.$, $\left.\sin \left(\frac{\pi}{n}\right)\right) . \quad M_{\lambda_{i}}$ lies to one side of $P$ at $\left(\lambda, \cos \left(\frac{\pi}{n}\right), \sin \left(\frac{\pi}{n}\right)\right)$, thus $G$ cannot be branched at this point. Furthermore, from the geometry of $C_{\lambda_{i}}$, we see that the preimage set of $G\left(\left(\lambda, \cos \left(\frac{\pi}{n}\right), \sin \left(\frac{\pi}{n}\right)\right)\right)$ in $M_{\lambda_{i}}$ consists only of the point $\left(\lambda, \cos \left(\frac{\pi}{n}\right), \sin \left(\frac{\pi}{n}\right)\right)$. Thus the degree of the covering map $G$ must be 1 . It follows that the total area of $\operatorname{Im}\left(M_{\lambda_{i}}\right)$ must be less than $2 \pi$ for all $i$, even when the area is counted with multiplicity. (In fact, the area is close to $\pi$ for large values of $\lambda_{i}$.) Therefore the total curvature of $M_{\lambda_{i}}$ is less than $2 \pi$ for all $i$, and the limit surface $\hat{M}_{\lambda}$ has total curvature at most $2 \pi$. In particular, $\hat{M}_{\lambda}$ has finite total curvature.

Since conjugation is an isometry, we know that $\tilde{M}_{\lambda}$ also has finite total curvature. First we extend $\tilde{M}_{\lambda}$ by reflection through the plane containing $\tilde{\alpha}_{1}$, and then we extend further by reflection through the plane containing $\tilde{\alpha}_{4}$. The resulting surface is an annulus with one complete end. This end has finite total curvature. Thus we can apply the result of Schoen [Scn] to conclude that this end must be either a planar end or a catenoid end. Clearly $\hat{M}_{\lambda}$ does not have a planar end, since the rays $\alpha_{1}$ and $\alpha_{4}$ do not lie in a common plane. We conclude that $\hat{M}_{\lambda}$ has a helicoid end.

We now show that $\hat{M}_{\lambda}$ is the unique minimal surface with a helicoid end and boundary $\hat{C}_{\lambda}$, that is a graph over $D$. Assume there is another such surface $S$. Consider sliding $S$ underneath $\hat{M}_{\lambda}$; that is, consider the proof of the last claim, but with " $M_{\lambda_{k}}$ " replaced by " $S$ " and " $M_{\lambda_{j}}$ " replaced by " $\hat{M}_{\lambda}$ ". If $S$ is slid underneath $\hat{M}_{\lambda}$ in this way, contact "at infinity" (meaning contact at the ends) between $\hat{M}_{\lambda}$ and a copy of $S$ cannot occur before the boundaries coincide. This follows, since by assumption $S$ has a helicoid end, and thus the ends of $S$ and $\hat{M}_{\lambda}$ are asymptotic to each other. Also, by the interior maximum principle, contact at a finite point cannot occur before the boundaries coincide. So first contact occurs exactly when the boundaries coincide, and therefore $S$ lies underneath $\hat{M}_{\lambda}$. Similarly, we can show that 


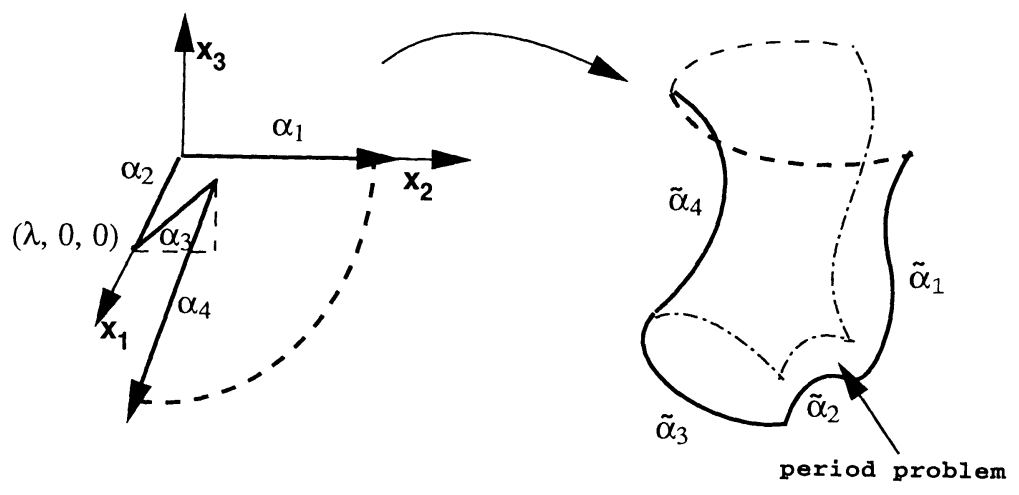

Figure 3.7: The surface $\hat{M}_{\lambda}$ and its conjugate surface $\tilde{M}_{\lambda}$.
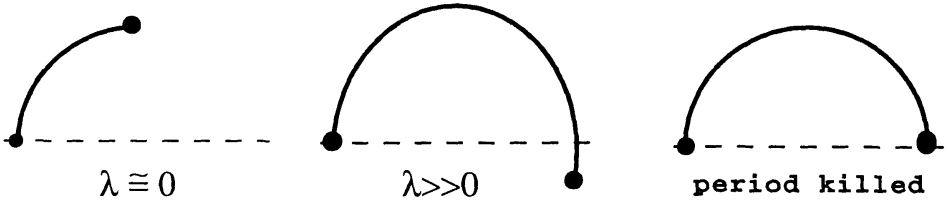

Figure 3.8: The planar geodesic $\tilde{\alpha}_{3}$ lying in a plane: for $\lambda$ close to zero; for $\lambda$ large; and for $\lambda$ adjusted to kill the period

$\hat{M}_{\lambda}$ lies underneath $S$, and we conclude $S=\hat{M}_{\lambda}$.

Since $\tilde{M}_{\lambda}$ is the conjugate surface of a graph over a convex domain, it is also a graph $[\mathbf{K r}]$, and is therefore embedded. Also, since $\tilde{M}_{\lambda}$ is the conjugate of a surface with a single end that is a 90 degree arc of a helicoid end, $\tilde{M}_{\lambda}$ itself has a single end that is a 90 degree arc of a catenoid end. If $\tilde{\alpha}_{2}$ and $\tilde{\alpha}_{4}$ were to lie in the same plane, then $\tilde{M}_{\lambda}$ could be extended by Schwarz reflection to a complete embedded minimal surface with catenoid ends. (The Schwarz reflection principle states that a minimal surface, which meets a plane orthogonally, may be extended to a larger minimal surface by reflection in that plane $[\mathbf{S c z}],[\mathbf{H o M e}]$.) This extended surface would be an $n$-oid with a handle symmetrically placed in the middle, i.e. an $n$-oid of genus 1 . However, $\tilde{\alpha}_{2}$ and $\tilde{\alpha}_{4}$ do not necessarily lie in the same plane. This period problem can be viewed clearly by considering $\tilde{\alpha}_{3}$ lying in a plane (see Figure 3.8).

As $\lambda \rightarrow 0$, the length of $\tilde{\alpha}_{2}$ approaches zero and $\tilde{\alpha}_{3}$ approaches a curve on the fundamental piece of the $n$-oid, as in the first part of Figure 3.8. We wish to show that for some large value of $\lambda, \tilde{\alpha}_{3}$ appears as in the second part of Figure 3.8. Then, by the Intermediate Value theorem, there will exist a value of $\lambda$ for which $\tilde{\alpha}_{3}$ appears as in the third part of Figure 3.8. Therefore 


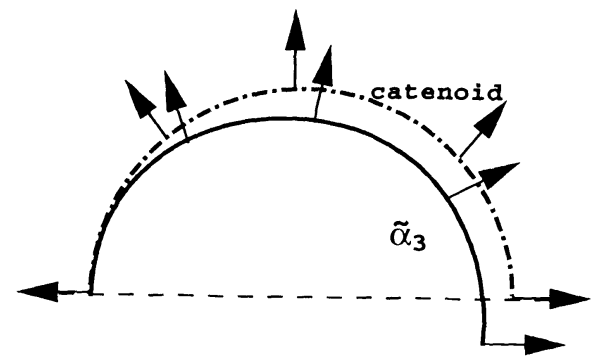

Figure 3.9: The planar geodesic $\tilde{\alpha}_{3}$ and a half-circle lying in the catenoid

the period problem will be solved.

We accomplish this with a helicoidal comparison argument. Consider a half-turn of a helicoid slid on "top" (again with respect to $x_{1}$ as height) of $\hat{M}_{\lambda}$, so that they share the boundary $\alpha_{2}, \alpha_{3}$, and $\alpha_{4}$, and so that $\hat{M}_{\lambda}$ and this half-turn of a helicoid lie on the same side of the plane $P$ through the points $(0,0,0),(\lambda, 0,0)$, and $\left(\lambda, \cos \left(\frac{\pi}{n}\right), \sin \left(\frac{\pi}{n}\right)\right)$. If $\lambda$ is sufficiently large, this "sliding" can be done so that the first moment of contact occurs along the boundary curves of the two surfaces. We note that the normal vectors of the helicoid and $\hat{M}_{\lambda}$ coincide at $(\lambda, 0,0)$ and $\left(\lambda, \cos \left(\frac{\pi}{n}\right), \sin \left(\frac{\pi}{n}\right)\right)$, but not, by the boundary point maximum principle, at any point of the interior of $\alpha_{3}$. Since $\hat{M}_{\lambda}$ lies below the helicoid, the normal of $M_{\lambda}$ must turn faster than the normal of the helicoid, when moving from $\left(\lambda, \cos \left(\frac{\pi}{n}\right), \sin \left(\frac{\pi}{n}\right)\right)$ to $(\lambda, 0,0)$ along $\alpha_{3}$. The same is then true along the corresponding curves in $\tilde{M}_{\lambda}$ and the conjugate surface to the helicoid. Recall that the catenoid is the conjugate surface to the helicoid, thus, the conjugate of $\alpha_{3}$, as a curve on the helicoid, is a half-circle. Since the conjugate map is an isometry, the conjugate of $\alpha_{3}$ as a curve on $\hat{M}_{\lambda}$, and as a curve on the helicoid, must be of equal length. The curve $\tilde{\alpha}_{3}$ is thus forced to lie below the half-circle in the catenoid (see Figure 3.9).

Remark. In order to fully justify the picture we have given of the genus-1 symmetric $n$-oid, we will show that the only branch points of this surface are simple branch points on curves identified, under reflection, with the curve $\tilde{\alpha}_{1}$.

First note that the Gauss map must be $n$-to- 1 on the genus- $1 n$-oid. This follows from a result of Jorge-Meeks [JoMe]: if the $n$ ends of a minimal surface are each separately embedded, then the degree of the Gauss map is $g+n-1$, where $g$ is the genus of the underlying Riemann surface.

It is shown in the proof above that $\lambda$ can be chosen so that $\tilde{M}_{\lambda}$ has no period problem. Choose this value for $\lambda$. Note that $\hat{M}_{\lambda}$ has the same normal 
vector at the two extremes of $\alpha_{1}$ : one of these extremes is the endpoint $(0,0,0)$; and the other extreme is the limit as one travels out to $\infty$ along the positive $x_{2}$-axis. It follows that the Gauss map must turn back on itself at some point on $\alpha_{1}$, hence the Gauss map has a branch point at some point on $\alpha_{1}$. This fact enables us to locate $2 n$ branch points of the Gauss map on the resulting symmetric $n$-oid of genus 1 . From the Riemann-Hurwitz formula, we see that these are the only branch points and they must all be simple.

Remark. Theorem 3.1 will not hold in the case $n=2$. In this case $\alpha_{3}$ is parallel to the $x_{3}$-axis and therefore the half turn of the helicoid can be slid underneath $\hat{M}_{\lambda}$ for all positive values of $\lambda$. Then by helicoidal comparison we will always have a situation comparable to the one pictured in figure 3.9 , except that $\tilde{\alpha}_{3}$ will sit above the half circle of the catenoid, thus the period cannot be killed. Of course, Schoen [Scn] has shown that the only immersed minimal surfaces with two catenoid ends (and no other ends) are the catenoids themselves.

A natural question to ask is whether one can add more handles to the $n$-oid, especially while preserving symmetry. Our next result shows that a certain example is impossible.

Theorem 3.3. There does not exist a symmetric $n$-iod of genus-n such that one handle is situated on each of the rays originating at the center of the $n$-iod and directed through the center of each of the catenoid ends.

Proof. Assume the surface exists, and let $M$ be the conjugate of a fundamental piece of the surface. Let $\tilde{M}$ be the conjugate surface of $M$ (see Figure 3.10). Regardless of the length of $\alpha_{3}$ in $M$, we can slide a piece of a helicoid underneath $M$ (again w.r.t. the $x_{1}$ direction), so that: the boundary of $M$ and the boundary of the helicoid-piece coincide along $\alpha_{1} \cup \alpha_{2} \cup \alpha_{3}$; $M$ and the helicoid-piece lie on the same side of the plane which contains $\alpha_{1} \cup \alpha_{2} \cup \alpha_{3}$; and the interior of $M$ lies strictly to one side of the interior of the helicoid-piece. Then moving from the origin along $\alpha_{2}$, the normal of $M$ must rotate ahead of the normal of the helicoid, forcing the corresponding curves in the conjugate surfaces to always appear as in Figure 3.9. Therefore the period cannot be killed.

Remark. Using Karcher's view of the $n$-oid as the limit of a deformation of the $2 n$-winged Scherk's towers ([Ka1], [Ka3]), Martin Traizet was able to numerically argue the existence of a symmetric $n$-oid of genus $n$ with handles situated on rays originating from the center and bisecting the axes of the catenoid ends [Tr] (see Figure 3.11). 


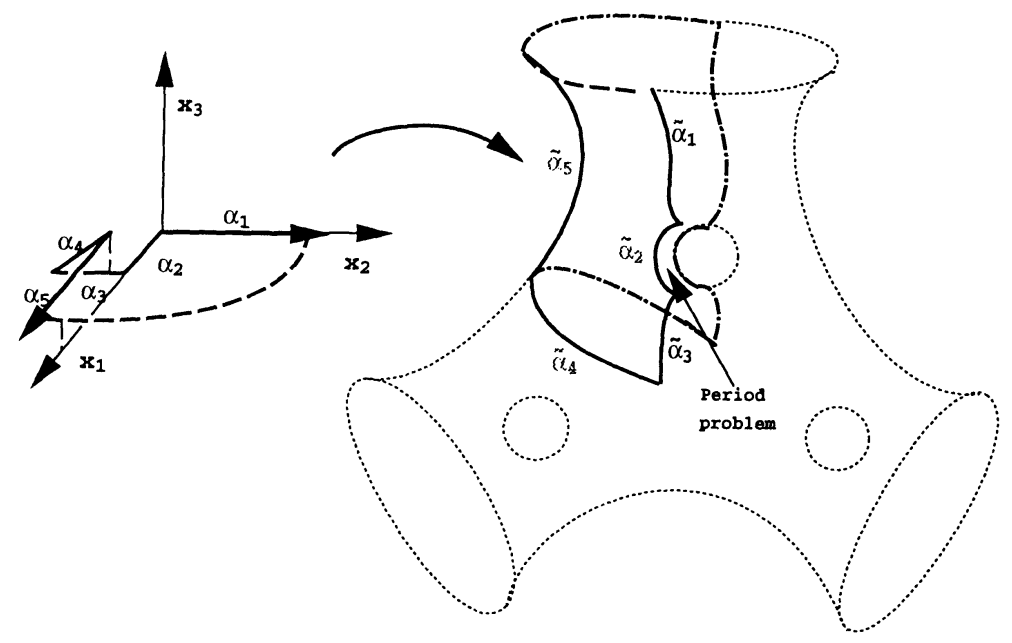

Figure 3.10: The surface $M$ and its conjugate surface $\tilde{M}$

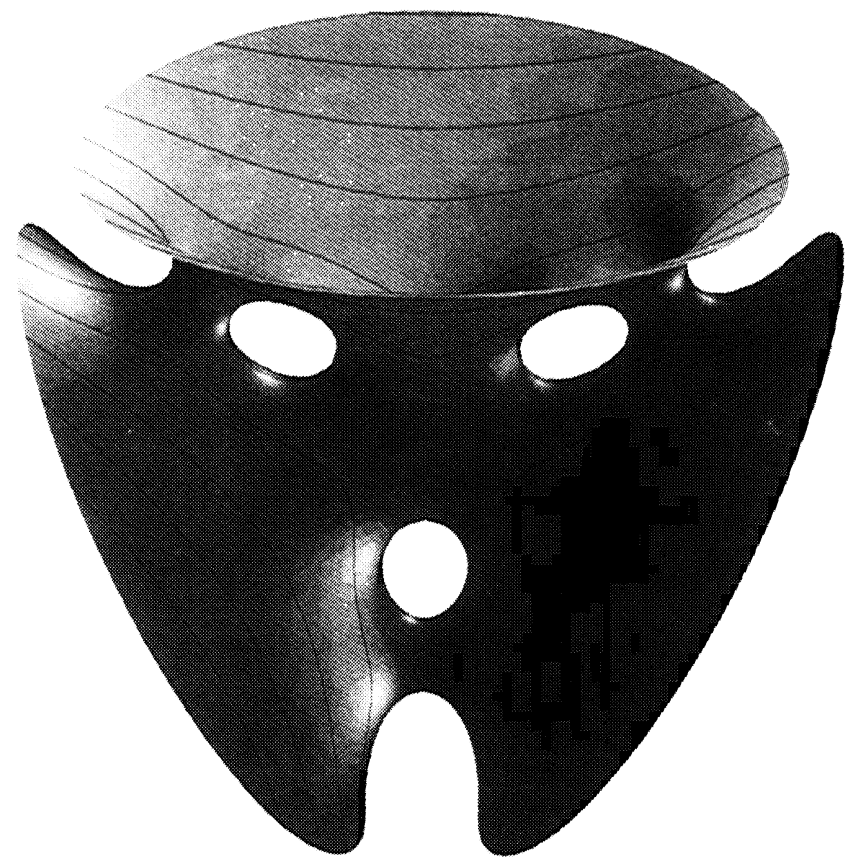

Figure 3.11: The trinoid of genus 3 

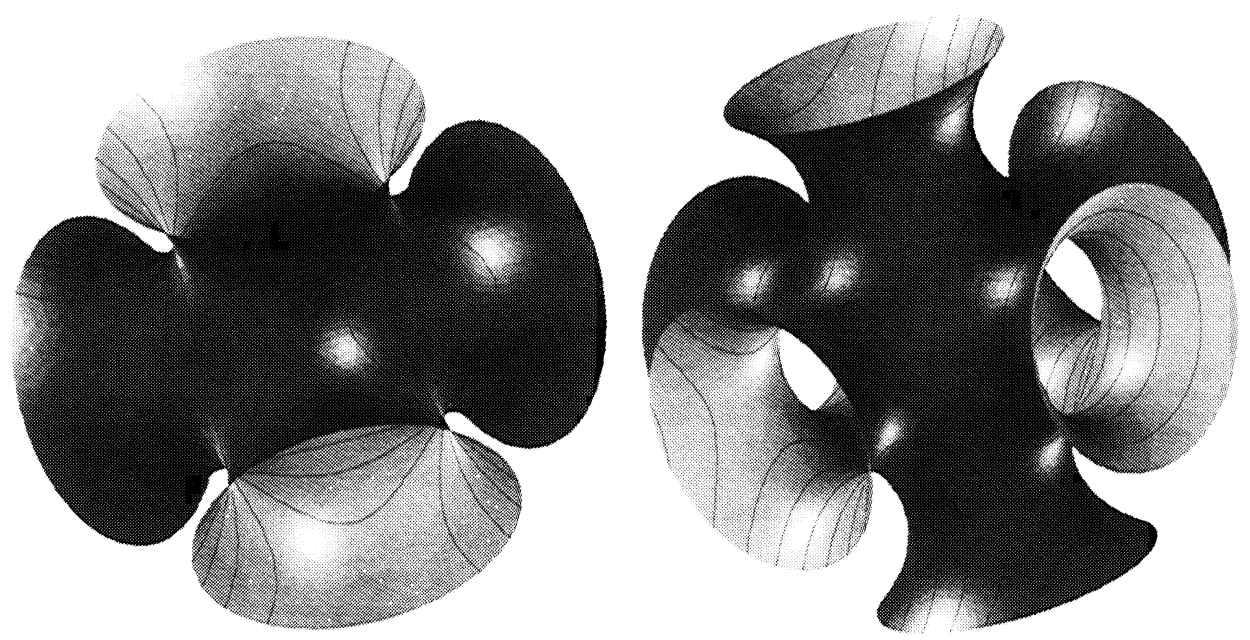

Figure 4.12: The tetroid and the octoid

\section{Minimal Surfaces Based on the Platonic Solids}

The method of the proof of Theorem 3.1 can be used to prove the existence of other immersed finite total curvature surfaces with catenoid ends and genus greater than zero. Recently Y. Xu $[\mathbf{X u}]$ constructed genus- 0 immersed minimal surfaces based on the Platonic solids. Topologically, they can be thought of as the surface of each Platonic solid with a catenoid end replacing each vertex of the solid (see Figure 4.12). As before, we rely on the existence of the genus zero surfaces to construct their higher genus counterparts.

Let $\mathcal{T}, \mathcal{C}, \mathcal{O}, \mathcal{D}$ and $\mathcal{I}$ be the symmetry groups of the Platonic solids: the tetrahedron, cube, octahedron, dodecahedron, and icosahedron, respectively. Note that $\mathcal{C}$ and $\mathcal{O}$ are isomorphic, as are $\mathcal{D}$ and $\mathcal{I}$.

Theorem 4.1. The following minimal surfaces with catenoid ends and finite total curvature exist:

1) A genus-3 surface with 4 ends and symmetry group isomorphic to $\mathcal{T}$.

2) A genus-5 surface with 8 ends and symmetry group isomorphic to $\mathcal{C}$.

3) A genus-7 surface with 6 ends and symmetry group isomorphic to $\mathcal{O}$ (see Figure 4.13).

4) A genus-11 surface with 20 ends and symmetry group isomorphic to $\mathcal{D}$.

5) A genus-19 surface with 12 ends and symmetry group isomorphic to $\mathcal{I}$. 


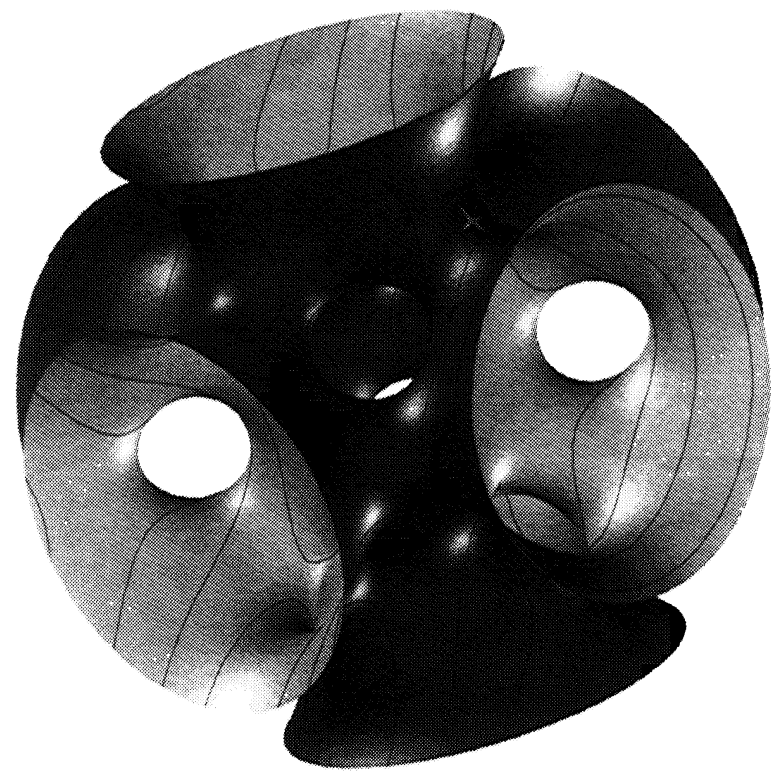

Figure 4.13: The octoid of genus 7

Proof. Quotienting by all symmetries, we have the fundamental piece, and its conjugate, of the genus-0 examples. They appear in Figure 4.14, where $\theta_{1}$, $\theta_{2}$ depend on which Platonic solid we consider. Consider the contour $C$ and its conjugate (see Figure 4.15). When projected onto a plane perpendicular to $\alpha_{2}$ and $\alpha_{4}$, the contour projects to part of the boundary of an unbounded convex domain. Viewing the direction of $\alpha_{4}$ as the height, the existence and uniqueness of a minimal graph with boundary $C$ and a helicoid end follow as in the proof of Theorem 3.1. Looking at the conjugate of this surface, we see that we have a single period problem. Again, since $\theta_{1}<\frac{\pi}{2}$, the helicoidal comparison argument shows that this period problem can be solved.

We list below the values of the angles $\theta_{1}$ and $\theta_{2}$ for each of the five surfaces:

1) tetroid $\theta_{1}=\frac{\pi}{3}, \theta_{2}=\frac{\pi}{3}$.

2) cuboid $\theta_{1}=\frac{\pi}{3}, \theta_{2}=\frac{\pi}{4}$.

3) octoid $\theta_{1}=\frac{\pi}{4}, \theta_{2}=\frac{\pi}{3}$.

4) dodecoid $\theta_{1}=\frac{\pi}{3}, \theta_{2}=\frac{\pi}{5}$.

5) icosoid $\theta_{1}=\frac{\pi}{5}, \theta_{2}=\frac{\pi}{3}$.

Remark. Xu also constructs minimal surfaces which can be thought of as Platonic solids with catenoid ends added to the edges. While in these cases we can show that the contour $C$ bounds a minimal surface, we find we can not apply the helicoidal comparison test to kill the period as the angle 

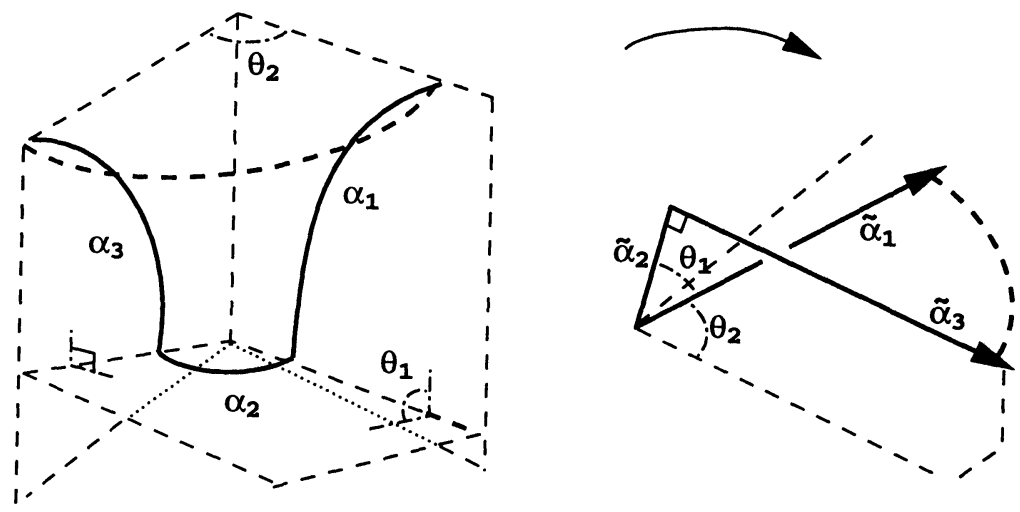

Figure 4.14: A fundamental piece of the genus-0 surface, and its conjugate surface

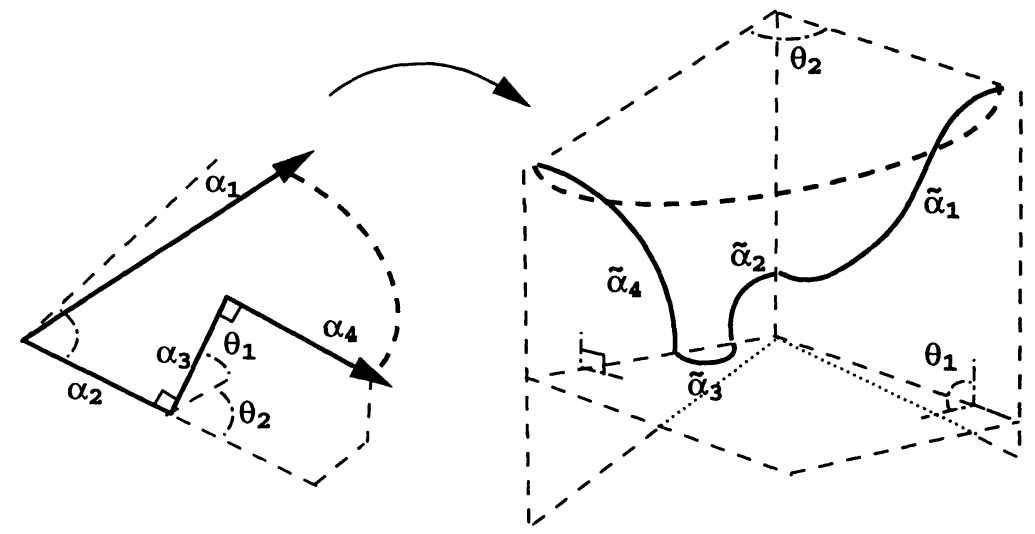

Figure 4.15: The fundamental piece with contour $C=\alpha_{1} \cup \alpha_{2} \cup \alpha_{3} \cup \alpha_{4}$, and its conjugate, which is a fundamental piece of the higher genus surface 

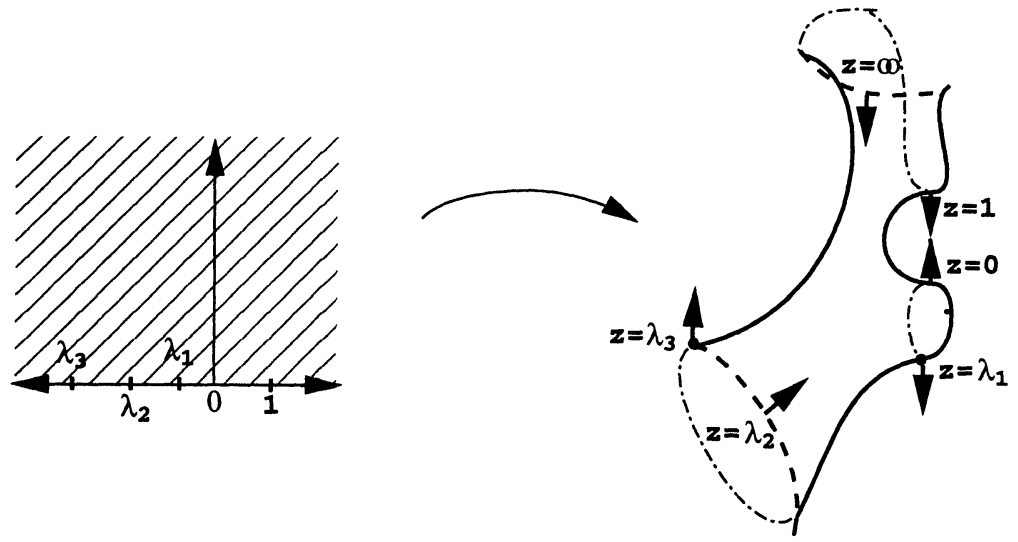

Figure 4.16: The image of the Weierstrass Data in equation (5.1)

between $\alpha_{2}$ and $\alpha_{3}$, as well as the angle between $\alpha_{3}$ and $\alpha_{4}$, is not $\frac{\pi}{2}$.

\section{Weierstrass Data for the Genus-1 Trinoid}

To construct Weierstrass data for the genus- 1 trinoid, it is convenient to consider one fourth of the surface obtained by quotienting by the reflection in $\mathcal{P}$ and the reflection in one other reflectional plane of symmetry.

Let $\bar{M}$ be $\{z \in \mathbb{C}: \operatorname{Im}(z) \geq 0\}$. Consider the Weierstrass data satisfying

$$
g^{2}=c \frac{(z-1)\left(z-\lambda_{1}\right)}{z\left(z-\lambda_{3}\right)^{2}}, \quad \eta=\frac{\left(z-\lambda_{3}\right) d z}{g\left(z-\lambda_{2}\right)^{2}},
$$

with $0>\lambda_{1}>\lambda_{2}>\lambda_{3}$. With this Weierstrass data, integrating over $\bar{M}$, we have the surface that is one fourth of the genus-1 trinoid (not necessarily fully symmetric), up to some period problems. The constant $c$ can be chosen so that the angle between the normals at the ends of the surface is $\frac{2 \pi}{3}$ (see Figure 4.16). The constant $c$ is a positive real, and its exact value is

$$
c=\frac{-3 \lambda_{2}\left(\lambda_{2}-\lambda_{3}\right)^{2}}{\left(\lambda_{2}-1\right)\left(\lambda_{2}-\lambda_{1}\right)} .
$$

To solve the period problems, we need to have the boundary planar geodesics $\alpha_{1}$ and $\alpha_{3}$ in the same plane; we also need to have $\alpha_{2}, \alpha_{4}$, and $\alpha_{5}$ all within a single plane. This can be accomplished by the proper choice of $\lambda_{1}, \lambda_{2}$, and $\lambda_{3}$. Using the MESH program [MESH] and a Simplex algorithm, we have found values for $\lambda_{i}$ so that the Weierstrass data produces one fourth of the symmetric genus- 1 trinoid. Surprisingly, we also found one other set of values for $\lambda_{i}$ which solves the period problem. This surface is not as symmetric, and suggests the existence of a larger family of less symmetric n-oids of genus 1 (see Figure 4.17). 

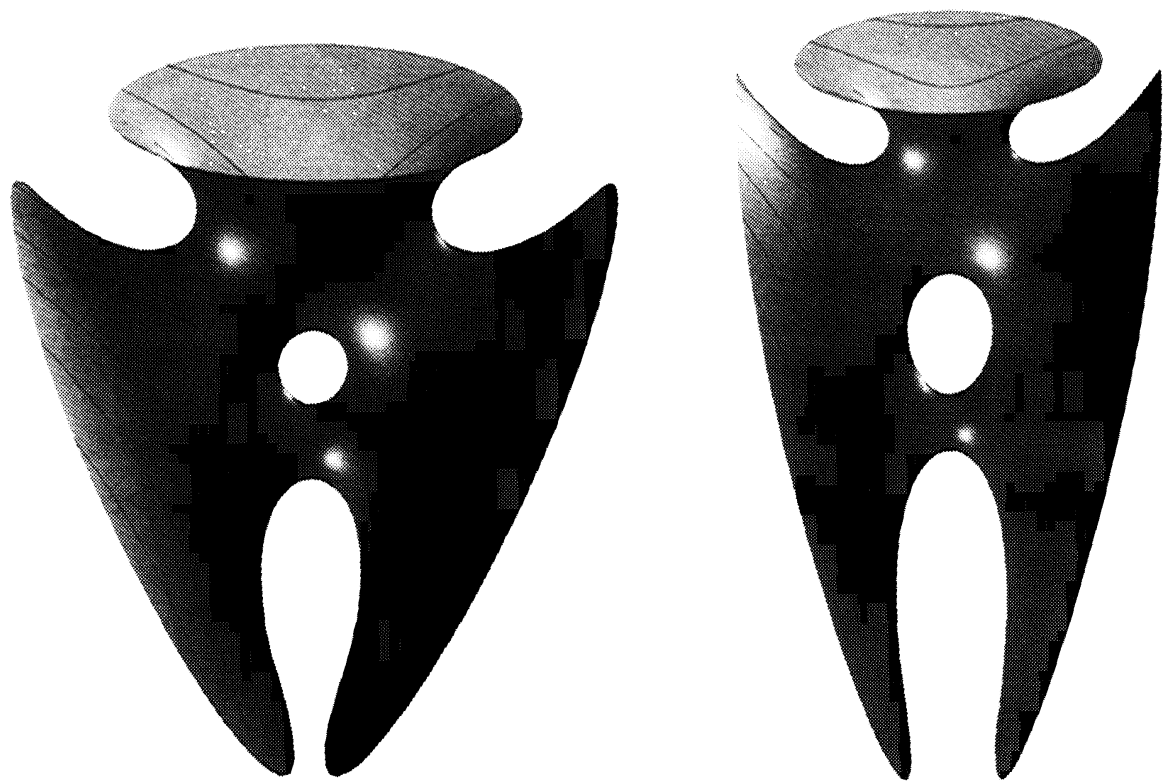

Figure 4.17: Less symmetric trinoids of genus 1: (left) with angles of $\frac{2 \pi}{3}$ between the normals of the ends, and (right) where those angles are $\frac{3 \pi}{5}, \frac{3 \pi}{5}$, and $\frac{4 \pi}{5}$

By adjusting the value of $c$ one can still solve the period problem with the Simplex method and produce genus- 1 trinoids where the normals at the ends to not form angles of $\frac{2 \pi}{3}$ (see Figure 4.17).

\section{References}

[HKR] D. Hoffman, H. Karcher, and H. Rosenberg, Embedded Minimal Annulv in $\mathbb{R}^{3}$ Bounded by a Pair of Stranght Lines, Commentarii Mathematici Helvetici, 66 (1991), 599-617.

[HoMe] D. Hoffman, W. H. Meeks III, Minımal Surfaces Based on the Catenoid, Amer. Math. Monthly, 97(8) (1990).

[JeSe] H. Jenkins and J. Serrin, Varaational Problems of Miminal Surface Type II. Boundary Value Problems for the Minimal Surface Equation, Arch. Rational Mech. Anal., 21 (1966), 321-342.

[JoMe] L. P. M. Jorge and W. H. Meeks III, The topology of complete minimal surfaces of finite total Gaussian curvature, Topology, 22 (2) (1983), 203-221.

[Ka1] H. Karcher, Embedded Minımal Surfaces Derived From Scherk's Surfaces, Manuscripta math., 62 (1988), 83-114.

[Ka2] H. Karcher, The Triply Periodic Minimal Surfaces of Alan Schoen and their Constant Mean Curvature Companions, Manuscripta math., 64 (1989), 291-357.

[Ka3] H. Karcher, Construction of Minimal Surfaces, Surveys in Geometry, 1-96, University of Tokyo, 1989 (Also: Lecture Notes No. 12, SFB256, Bonn, 1989).

[Ka4] H. Karcher, Construction of Higher Genus Embedded Minımal Surfaces, preprint. 
[Kr] R. Krust, The Conjugate of a Minimal Graph over a Convex Domain is a Graph, Personal communication between R. Krust and H. Karcher (see [Ka3]).

[MESH] J. Hoffman, Software for Constructing Minimal Surfaces Using Weierstrass Data. G.A.N.G. Lab, University of Massachussets, Amherst, MA 01003.

[MeYa] W. H. Meeks and S.-T. Yau, The Classical Plateau Problem and the Topology of Three-Dimensional Manifolds, Topology, 21(4) (1982), 409-442.

[Ni1] J. C. C. Nitsche, Uber ein verallgemeinertes Dirichletsches Problem fur die Minimalflachengleichung und hebbare Unstetigkeiten ihrer Losungen. Math. Ann., 158 (1965), 302-214.

[Ni2] J. C. C. Nitsche, Lectures on Minimal Surfaces, Vol. 1. Cambridge University Press, New York, NY, 1989.

[Scn] R. Schoen, Uniqueness, Symmetry, and Embeddedness of Minimal Surfaces, Journal of Differential Geometry, 18 (1982), 791-809.

[Scz] H. A. Schwarz, Gesammelte mathematische Abhandlungen. 2 vols, Springer, Berlin, 1890.

[Tr] M. Traizet, Personal communication.

[Xu] Y. Xu, Symmetric Minimal Surfaces. To appear in the Pacific Journal of Math.

Received April 19, 1993 and revised October 25, 1993.

UNIVERSITY OF MASSACHUSSETTS

AmHerst, MA 01003

E-mail address: berglund@smectos.gang.umass.edu

AND

Mathematical Institute

TOHOKU UNIVERSITY

SEndAI 980, JAPAN

E-mail address: wayne@smectos.gang.umass.edu 



\title{
PACIFIC JOURNAL OF MATHEMATICS
}

Founded by E. F. Beckenbach (1906-1982) and F. Wolf (1904-1989)

\section{EDITORS}

\author{
Sun-Yung Alice Chang (Managing Editor) \\ University of California \\ Los Angeles, CA 90095-1555 \\ pacific@math.ucla.edu
}

\section{F. Michael Christ}

University of California

Los Angeles, CA 90095-1555

christ@math.ucla.edu

Thomas Enright

University of California

San Diego, La Jolla, CA 92093

tenright@ucsd.edu

Nicholas Ercolani

University of Arizona

Tucson, AZ 85721

ercolani@math.arizona.edu
Robert Finn

Stanford University

Stanford, CA 94305

finn@gauss.stanford.edu

Vaughan F. R. Jones

University of California

Berkeley, CA 94720

vfr@math.berkeley.edu

Steven Kerckhoff

Stanford University

Stanford, CA 94305

spk@gauss.stanford.edu
Martin Scharlemann

University of California

Santa Barbara, CA 93106

mgscharl@math.ucsb.edu

Gang Tian

Courant Institute

New York University

New York, NY 10012-1100

tiang@taotao.cims.nyu.edu

V. S. Varadarajan

University of California

Los Angeles, CA 90095-1555

vsv@math.ucla.edu

\section{SUPPORTING INSTITUTIONS}

\section{CALIFORNIA INSTITUTE OF TECHNOLOGY \\ NEW MEXICO STATE UNIVERSITY \\ OREGON STATE UNIVERSITY \\ STANFORD UNIVERSITY \\ UNIVERSITY OF ARIZONA \\ UNIVERSITY OF BRITISH COLUMBIA \\ UNIVERSITY OF CALIFORNIA UNIVERSITY OF HAWAII}

\author{
UNIVERSITY OF MONTANA \\ UNIVERSITY OF NEVADA, RENO \\ UNIVERSITY OF OREGON \\ UNIVERSITY OF SOUTHERN CALIFORNIA \\ UNIVERSITY OF UTAH \\ UNIVERSITY OF WASHINGTON \\ WASHINGTON STATE UNIVERSITY
}

The supporting Institutions listed above contribute to the cost of publication of this Journal, but they are not owners or publishers and have no responsibility for its contents or policies.

Manuscripts must be prepared in accordance with the instructions provided on the inside back cover.

The Pacific Journal of Mathematics (ISSN 0030-8730) is published monthly except for July and August. Regular subscription rate: $\$ 215.00$ a year (10 issues). Special rate: $\$ 108.00$ a year to individual members of supporting institutions.

Subscriptions, orders for back issues published within the last three years, and changes of subscribers address should be sent to Pacific Journal of Mathematics, P.O. Box 4163, Berkeley, CA 94704-0163, U.S.A. Prior back issues are obtainable from Kraus Periodicals Co., Route 100, Millwood, NY 10546.

The Pacific Journal of Mathematics at the University of California, c/o Department of Mathematics, 981 Evans Hall, Berkeley, CA 94720 (ISSN 0030-8730) is published monthly except for July and August. Second-class postage paid at Berkeley, CA 94704, and additional mailing offices. POSTMASTER: send address changes to Pacific Journal of Mathematics, P.O. Box 6143, Berkeley, CA 94704-0163.

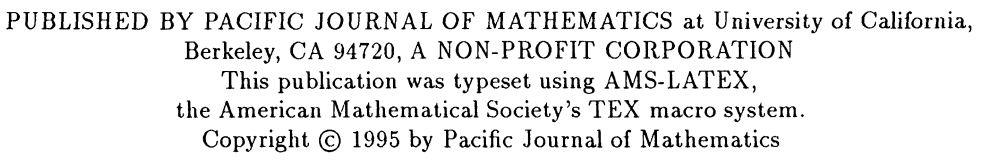




\section{PACIFIC JOURNAL OF MATHEMATICS}

\section{Volume $171 \quad$ No. $2 \quad$ December 1995}

On $H^{p}$-solutions of the Bezout equation

ERIC AMAR, JOAQUIM BRUNA FLORIS and ARTUR NiCOLAU

Amenable correspondences and approximation properties for von Neumann algebras

309

Claire ANANTHARAman-DElaroche

On moduli of instanton bundles on $\mathbb{P}^{2 n+1}$

VinCENZO ANCONA and Giorgio MaRia OtTAVIANI

Minimal surfaces with catenoid ends

JORGEN BERGLUND and WAYNE ROSSMAN

Permutation model for semi-circular systems and quantum random walks

PHILIPPE BIANE

The Neumann problem on Lipschitz domains in Hardy spaces of order less than one

RUSSELL M. BROWN

Matching theorems for twisted orbital integrals

409

REBECCA A. HERB

Uniform algebras generated by holomorphic and pluriharmonic functions on strictly

pseudoconvex domains

ALEXANDER IzZO

Quantum Weyl algebras and deformations of $U(g)$

NAIHUAN JING and JAMES ZHANG

Calcul du nombre de classes des corps de nombres

STÉPHANE LOUBOUTIN

On geometric properties of harmonic Lip ${ }_{1}$-capacity

PertTi MatTila and P. V. PARAmonov

Reproducing kernels and composition series for spaces of vector-valued holomorphic functions

Bent ØRsted and Genkai Zhang

Iterated loop modules and a filtration for vertex representation of toroidal Lie algebras

S. ESWARA RAO

The intrinsic mountain pass

MARTIN SCHECHTER

A Frobenius problem on the knot space

RON G. WANG

On complete metrics of nonnegative curvature on 2-plane bundles

DAVID YANG

Correction to: "Free Banach-Lie algebras, couniversal Banach-Lie groups, and more"

VLADIMIR G. PESTOV

Correction to: "Asymptotic radial symmetry for solutions of $\Delta u+e^{u}=0$ in a punctured disc"

KAI Seng (KAISing) CHOU (Tso) and Tom YAU-Heng WAN 